\title{
Advances in thermosonication for the inactivation of endogenous enzymes in foods
}

\begin{abstract}
The main objective of food pasteurization is to inactivate pathogens and reduce spoilage organisms. For certain foods (e.g. orange juice) and in particular with emerging non-thermal technologies such as ultrasound and high pressure processing, endogenous and deteriorative enzymes can be very resistant. Therefore, enzymes should also be used as pasteurization references to avoid spoilage of processed foods during storage. Thus, endogenous food enzymes should be inactivated to increase its shelf life. In this chapter a review of the effects of thermo-sonication (simultaneous ultrasound and heat) on food enzymes was carried out. The ultrasound treatment denatures the enzyme, resulting in enzyme conformational changes and decrease in its activity. A variety of foods has been processed by ultrasound without heat, thermos-sonication and mano-thermo-sonication and the effect on enzymes is reviewed. Thermo-sonication and mano-thermo-sonication are recommended for enzyme inactivation, since ultrasound at room temperature is often ineffective against most food enzymes. The most relevant plant endogenous enzymes are pectinmethylesterase (PME), polygalacturonase (PG), polyphenoloxidase (PPO) and peroxidase (PRO), which are important for fruit and vegetable products. In addition lactoperoxidase (LPO), $\gamma$-glutamyltranspeptidase (GTP) and alkaline phosphatase (AP) from milk and dairy products are also reviewed
\end{abstract}

Keyword: Dairy products; Enzyme; Fruit products; Pasteurization; Sonication; Ultrasound 